\section{PPE you can trust}

Initial Medical are specialists in infection control, offering practical, effective, and high-quality solutions. They also supply PPE, ideal for those working in all medical, healthcare, and aesthetic environments, including dental practices.

The range includes disposable surgical face masks, as well as gloves in vinyl, latex and nitrile.

Initial Medical can also help you with the correct storage, disposal and removal of clinical waste, including PPE that may have been contaminated with Coronavirus from a patient who is a confirmed or suspected carrier.

PPE is an essential element of infection control. Get it right to reassure your patients and your team of your commitment to their safety.

Initial Medical is helping a wide range of dental and other customers to get their businesses back up and running again, safely. For further information visit initial. co.uk/medical or call 08708504045 .

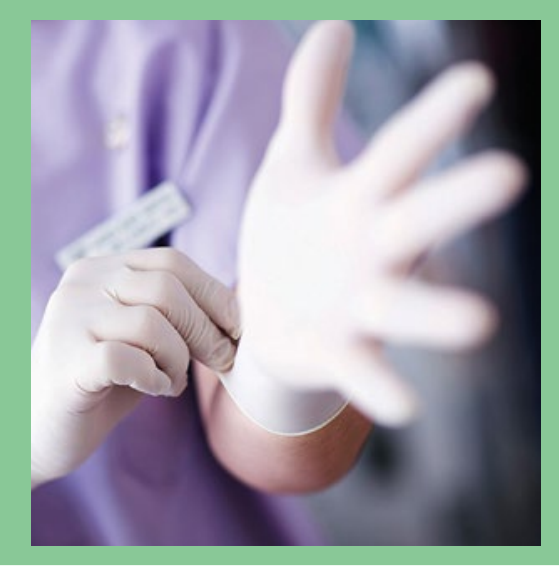

\title{
Reducing the risk of aerosol contamination
}

We have always known that unchecked dental aerosols can present a health risk, and the new Coronavirus strain has added an extra dimension, as it can be detected in the area of the mouth, nose and throat.

Of course, almost all dental instruments used in common treatments generate aerosols: low- and high-speed handpieces, turbines, sonic and ultrasonic devices, air water syringes, and air polishing devices.

Guided Biofilm Therapy (GBT), the systematic, predictable solution for dental biofilm management in professional prophylaxis uses cutting-edge air flow technologies, which also generate aerosols.

However, there are measures that can be put in place to reduce the risks to team members and patients alike.

Wearing the right PPE is key to keeping both staff and patients safe, so the correct use of mouth-nose protection masks, goggles, and face-shields, as well as the efficient and safe sterilisation of instruments is mandatory.

For example, AIRFLOW and PIEZON handpieces and all Instruments (PS) should be sterilised after each treatment. The surface disinfection and regular rinsing of the suction system are further obligatory measures.

Good ventilation of the treatment rooms after each patient is strongly recommended. ${ }^{1}$ Indeed, the use of a high-vacuum suction system with a suction volume of at least $300 \mathrm{l} / \mathrm{min}$, in combination with an optimal saliva ejector, can effectively reduce aerosol formation., ${ }^{2,3}$

In addition, four-handed dentistry allows optimal suction. ${ }^{4}$ When working two-handed for professional mechanical biofilm removal, a skilled suction technique with a high vacuum suction in combination with Optragate supports reduction of aerosols very well.

It has also been suggested that hydrogen peroxide $(\mathrm{H} 2 \mathrm{O} 2)$ at a minimum concentration of $0.5 \%$ or more is proven to effectively kill viruses. ${ }^{5,6}$

The new Coronavirus strain, SARSCoV-2, will be with us for a long time - and so will biofilm. We hope that you will soon be able to offer your patients a good feeling with the EMS Guided Biofilm Therapy.

For further details about how EMS Dental can support dental professionals in the UK moving forward visit www.ems-dental.com

\section{References}

1. Izzetti R, Nisi M, Gabriele M, Graziani. COVID19 Transmission in Dental Practice: Brief Review of Preventive Measures in Italy. J Dent Res 2020; 99: 1030-1038.

2. Graetz C, Bielfeldt J, Tillner A, Plaumann A, Dörfer C E. Splatter contamination in dental practices - how can it be prevented? Rev Med Chir Soc Med Nat lasi 2014; 118: 1122-1134.

3. Reitemeier B, Jatzwauk L, Jesinghaus S, Reitemeier C, Neumann K. Effektive Reduktion des Spraynebel-Rückpralls - Möglichkeiten und Grenzen. ZMK; 26: 662-673

4. Meng L, Hua F, Bian Z. Coronavirus Disease 2019 (COVID-19): Emerging and Future Challenges for Dental and Oral Medicine. J Dent Res 2020; 99: 481-487.

5. Caruso A A, Del Prete A, Lazzarino A I, Capaldi $\mathrm{R}$, Grumetto L. May hydrogen peroxide reduce the hospitalization rate and complications of SARS-CoV-2 infection? Infect Control Hosp Epidemiol 2020; 22: 1-5.

6. Mentel R, Shirrmakher R, Kevich A, Dreǐzin RS, Shmidt I. Virus inactivation by hydrogen peroxide. Vopr Virusol 1977; 6: 731-733.

\section{At the cutting-edge}

ClearCorrect from the Straumann Group is dentist-friendly by nature, having been developed from feedback and advice provided by clinicians. The clear aligners feature smooth, unscalloped trim lines that extend $1 \mathrm{~mm}$

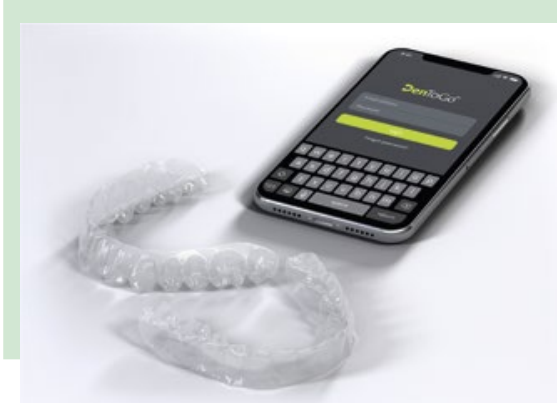

over the gingiva to significantly improve retention and reduce the need for engagers (attachments). ${ }^{1}$ They also come with a choice of either the Flex (pay-as-you-go) or Unlimited (as many aligners and retainers as needed for 5 years) schemes for maximum cost-efficiency.

The innovative DenToGo uses artificial intelligence to facilitate remote treatment monitoring, reducing the need for appointments while ensuring high quality treatment. It also helps to motivate patients and keep them engaged in their treatment for greater compliance.
Available with a comprehensive digital workflow and support that is second-tonone, ClearCorrect is the ideal solution for any clinician committed to operating at the cutting-edge of orthodontics.

For more information on ClearCorrect, visit https://www.straumann.com/ clearcorrect/en/home.html.

References

1. Cowley D P, Mah J, O'Toole B. The effect of gingival-margin design on the retention of thermoformed aligners. J Clin Ortho 2012; 46: 697-702. 